\title{
COUNTEREXAMPLES TO CONVEXITY OF $k$-INTERSECTION BODIES
}

\author{
VLADYSLAV YASKIN \\ (Communicated by Alexander Iosevich)
}

\begin{abstract}
It is a well-known result due to Busemann that the intersection body of an origin-symmetric convex body is also convex. Koldobsky introduced the notion of $k$-intersection bodies. We show that the $k$-intersection body of an origin-symmetric convex body is not necessarily convex if $k>1$.
\end{abstract}

\section{INTRODUCTION}

A body in $\mathbb{R}^{n}$ is a compact set with a non-empty interior. We say that a body $K$ is a star body if it is star-shaped about the origin and its radial function defined by

$$
\rho_{K}(\xi)=\max \{\lambda>0: \lambda \xi \in K\}, \quad \text { for } \xi \in S^{n-1},
$$

is positive and continuous.

The Minkowski functional of a star body $K$ is given by

$$
\|x\|_{K}=\min \{\lambda \geq 0: x \in \lambda K\}, \quad \text { for } x \in \mathbb{R}^{n} .
$$

It is easy to see that the latter is a homogeneous function of degree 1 on $\mathbb{R}^{n}$ and $\|\xi\|_{K}=\rho_{K}^{-1}(\xi)$, when $\xi \in S^{n-1}$.

The notion of the intersection body of a star body was introduced by Lutwak $[\mathrm{L}]$ in 1988 and has played an important role in Convex Geometry since then. The intersection body of a star body $K$ is defined to be a star body $I K$ whose radial function is given by

$$
\rho_{I K}(\xi)=\operatorname{vol}_{n-1}\left(K \cap \xi^{\perp}\right), \quad \text { for } \xi \in S^{n-1},
$$

where $\xi^{\perp}$ stands for the hyperplane $\left\{x \in \mathbb{R}^{n}:\langle x, \xi\rangle=0\right\}$.

Intersection bodies were a key ingredient in the solution of the celebrated Busemann-Petty problem. Let $K$ and $L$ be origin-symmetric convex bodies in $\mathbb{R}^{n}$ such that

$$
\operatorname{vol}_{n-1}\left(K \cap \xi^{\perp}\right) \leq \operatorname{vol}_{n-1}\left(L \cap \xi^{\perp}\right), \quad \text { for all } \xi \in S^{n-1} .
$$

Does it necessarily follow that

$$
\operatorname{vol}_{n}(K) \leq \operatorname{vol}_{n}(L) ?
$$

The answer to the problem is affirmative if $n \leq 4$ and negative if $n \geq 5$; see GKS, [K2], [Z2] for historical details.

A generalization of the original Busemann-Petty problem to sections of other dimensions is often called the lower-dimensional Busemann-Petty problem. Let

Received by the editors February 3, 2013.

2010 Mathematics Subject Classification. Primary 52A20.

Key words and phrases. Convex bodies, star bodies, $k$-intersection bodies.

This research was supported in part by NSERC. 
$1 \leq k \leq n-1$ be an integer and let $G(n, k)$ be the Grassmannian of all $k$-dimensional subspaces of $\mathbb{R}^{n}$. Let $K$ and $L$ be origin-symmetric convex bodies in $\mathbb{R}^{n}$ such that

$$
\operatorname{vol}_{k}(K \cap H) \leq \operatorname{vol}_{k}(L \cap H), \quad \text { for all } H \in G(n, k) .
$$

Is it true that

$$
\operatorname{vol}_{n}(K) \leq \operatorname{vol}_{n}(L) ?
$$

It is shown by Bourgain and Zhang [BZ] that the answer to this problem is negative if $k \geq 4$. Another proof is given in [K1. The cases $k=2$ and $k=3$ are still open in dimensions $n \geq 5$.

Related to this problem are certain classes of bodies that generalize the notion of the intersection body. One generalization is due to Zhang [Z1 and another to Koldobsky [K1]. In this paper we will only discuss Koldobsky's $k$-intersection bodies. For the relation between the two generalizations and other results see Milman's works [M1 and M2. Let us emphasize that the study of these classes of bodies is important for the understanding of the open cases of the lower-dimensional Busemann-Petty problem.

Let $1 \leq k \leq n-1$ and let $K$ and $L$ be origin-symmetric star bodies in $\mathbb{R}^{n}$. We say that $K$ is the $k$-intersection body of $L$ if for every $(n-k)$-dimensional subspace $H \subset \mathbb{R}^{n}$ we have

$$
\operatorname{vol}_{k}\left(K \cap H^{\perp}\right)=\operatorname{vol}_{n-k}(L \cap H) .
$$

One can see that 1-intersection bodies coincide (up to a scaling factor) with Lutwak's intersection bodies.

Koldobsky has shown that if $K$ is the $k$-intersection body of $L$, then the following relation holds (see e.g. [K2, Lemma 4.5]):

$$
\|x\|_{K}^{-k}=\frac{k}{(n-k)(2 \pi)^{k}}\left(\|\cdot\|_{L}^{-n+k}\right)^{\wedge}(x), \quad x \in \mathbb{R}^{n} \backslash\{0\},
$$

where in the right-hand side we have a Fourier transform in the sense of distributions; see next section for details. The latter formula implies, in particular, that for some bodies $L$ the corresponding $k$-intersection bodies may not exist when $k>1$.

Formula (1) can be used as a definition of $k$-intersection bodies in the case when $k$ is not necessarily an integer (and in fact, this is related to the concept of a space being embedded in $L_{-k}$; see [K2, Section 6.3]). For example, formula (11) for $0<k<1$ can be written as follows:

$$
\|x\|_{K}^{-k}=c_{n, k} \int_{S^{n-1}}|\langle x, \theta\rangle|^{-k}\|\theta\|_{L}^{-n+k} d \theta .
$$

Such bodies naturally arise in the theory of valuations, and in the following form

$$
\|x\|_{K}^{p}=\int_{L}|\langle x, \theta\rangle|^{p} d \theta, \quad(p>-1, p \neq 0),
$$

they are usually called the $L_{p}$-intersection bodies (see $\left.[\mathrm{H}], \mathrm{HL}\right]$ ). Note that with a different normalization these bodies are also known as polar $p$-centroid bodies (see e.g. [GG], LYZ, [LZ, [YY]).

There is a natural notion of the complex intersection body. Such bodies were introduced and studied by Koldobsky, Paouris and Zymonopoulou, KPZ2. (See also KKZ, where these studies were initiated.) It is shown that an origin-symmetric complex star body $K$ in $\mathbb{R}^{2 n}$ (which is naturally identified with $\mathbb{C}^{n}$ ) is a complex intersection body if and only if it is a 2 -intersection body in $\mathbb{R}^{2 n}$ and has certain rotational symmetries. 
It is a classical theorem of Busemann (see [G, Theorem 8.1.10] for example) that the intersection body of an origin-symmetric convex body is also convex. There are various generalizations and modifications of this result; see e.g. [MP, $[\mathrm{B}], \mathrm{KYZ}$. In particular, Berck $[\mathrm{B}]$ has shown that the $L_{p}$-intersection bodies of origin-symmetric convex bodies are convex for $p>-1, p \neq 0$. Until now it was unknown whether $k$-intersection bodies of origin-symmetric convex bodies are convex. The question was raised during discussions at various conferences; most recently Bernig asked this question at the Oberwolfach workshop on Convex Geometry and its Applications (December, 2012). In this paper we answer it in the negative for all $k=2,3, \ldots$, $n-1$.

It is worth noting that, in contrast with our result, Koldobsky, Paouris and Zymonopoulou [KPZ2] have shown that the complex intersection body of a complex convex body is convex (in other words, the 2-intersection body of a convex body in $\mathbb{R}^{2 n}$ with certain symmetries is necessarily convex). Thus the complex structure in fact plays a crucial role in preserving convexity.

For other properties of $k$-intersection bodies the reader is referred to [K2], [KPZ1, $[\mathrm{KY},[\mathrm{S}, \mathrm{Y}]$.

\section{TOOLS AND AUXILIARY RESUlTS}

Let $K$ be a star body. We say that $K$ is origin-symmetric if $\rho_{K}(\xi)=\rho_{K}(-\xi)$ for all $\xi \in S^{n-1}$. We would like to compute the Fourier transform of powers of $\|\cdot\|_{K}$, the Minkowski functional of $K$. Recall that, given a function $f \in L_{1}\left(\mathbb{R}^{n}\right)$, its Fourier transform $\widehat{f}$ is defined as follows:

$$
\widehat{f}(x)=\int_{\mathbb{R}^{n}} f(y) e^{-i\langle x, y\rangle} d y .
$$

Unfortunately, no power of $\|\cdot\|_{K}$ belongs to $L_{1}\left(\mathbb{R}^{n}\right)$. However, it is still possible to compute the desired Fourier transforms in the sense of distributions. Here we describe a basic idea; for details see [GS, [K2].

Let $\mathcal{S}\left(\mathbb{R}^{n}\right)$ be the Schwartz space of rapidly decreasing infinitely differentiable functions on $\mathbb{R}^{n}$. Elements of this space are referred to as test functions. Distributions are the elements of the dual space, $\mathcal{S}^{\prime}\left(\mathbb{R}^{n}\right)$, of linear continuous functionals on $\mathcal{S}\left(\mathbb{R}^{n}\right)$. The action of a distribution $f$ on a test function $\phi$ is denoted by $\langle f, \phi\rangle$. The Fourier transform of a distribution $f$ is defined to be a distribution $\hat{f}$ (we also use the notation $\left.(f)^{\wedge}\right)$ satisfying

$$
\langle\hat{f}, \phi\rangle=\langle f, \hat{\phi}\rangle
$$

for every test function $\phi$ from the space $\mathcal{S}\left(\mathbb{R}^{n}\right)$.

Let $K$ be a convex body and let $\xi \in S^{n-1}$. The parallel section function $A_{K, \xi}(t)$ is defined by

$$
A_{K, \xi}(t)=\operatorname{vol}_{n-1}\left(K \cap\left(\xi^{\perp}+t \xi\right)\right), \quad t \in \mathbb{R} .
$$

There is a remarkable connection between the derivatives of the parallel section function of a body $K$ and the Fourier transform of the powers of its Minkowski functional.

Theorem 2.1 (GKS, Theorem 1). Let $K$ be an origin-symmetric convex body in $\mathbb{R}^{n}$ with $C^{\infty}$ boundary, $k$ a non-negative integer, $k \neq n-1$, and $\xi \in S^{n-1}$.

(a) If $k$ is even, then

$$
\left(\|x\|_{K}^{-n+k+1}\right)^{\wedge}(\xi)=(-1)^{k / 2} \pi(n-k-1) A_{K, \xi}^{(k)}(0) .
$$


(b) If $k$ is odd, then

$$
\begin{aligned}
& \left(\|x\|_{K}^{-n+k+1}\right)^{\wedge}(\xi)=(-1)^{(k+1) / 2} 2(n-1-k) k ! \times \\
& \quad \times \int_{0}^{\infty} \frac{A_{K, \xi}(z)-A_{K, \xi}(0)-A_{K, \xi}^{\prime \prime}(0) \frac{z^{2}}{2}-\cdots-A_{K, \xi}^{(k-1)}(0) \frac{z^{k-1}}{(k-1) !}}{z^{k+1}} d z,
\end{aligned}
$$

where $A_{K, \xi}^{(k)}$ stands for the derivative of the order $k$ and the Fourier transform is considered in the sense of distributions.

In particular, it follows that for infinitely smooth bodies the Fourier transform of $\|x\|_{K}^{-n+k+1}$ restricted to the unit sphere is a continuous function (see also K2, Lemma 3.16]).

We also note that the previous theorem together with Brunn's theorem implies that for every origin-symmetric convex body $K$ the Fourier transforms of $\|x\|_{K}^{-n+2}$ and $\|x\|_{K}^{-n+3}$ are non-negative functions on the sphere (see [K2, Corollary 4.9]).

\section{Main Result}

Theorem 3.1. Let $k$ be an integer, $2 \leq k \leq n-1$. There is an origin-symmetric convex body $L$ in $\mathbb{R}^{n}$ such that its $k$-intersection body $K$ exists and is not convex.

Proof. We will consider three cases according to the value of $k: 4 \leq k \leq n-1$, $k=2, k=3$. The reader might have already noticed that the cases $k=2$ and $k=3$ usually differ from the rest. In our proof, the reason why we need a different construction for $k=2$ and $k=3$ is that the example used in Case 1 does not yield a convex body $L$ when $k=2$ or $k=3$.

Case 1. Let $4 \leq k \leq n-1$. For a small $\epsilon>0$ define an origin-symmetric star body $L=L_{\epsilon}$ by the formula:

$$
\|x\|_{L}^{-n+k}=|x|_{2}^{-n+k}-\epsilon^{k-1}(1-\epsilon)^{-n+k+1}\|x\|_{E}^{-n+k}, \quad x \in \mathbb{R}^{n} \backslash\{0\},
$$

where $|x|_{2}$ is the Euclidean norm and $E$ is the ellipsoid given by

$$
\|x\|_{E}=\left(\frac{x_{1}^{2}+\cdots+x_{n-1}^{2}}{(1-\epsilon)^{2}}+\frac{x_{n}^{2}}{\epsilon^{2}}\right)^{1 / 2} .
$$

Since $\|x\|_{E}^{-1}<|x|_{2}^{-1}$, it follows that $\|x\|_{L}^{-1}$ is positive for all $\epsilon>0$ small enough, and so the body $L$ is well defined.

We claim that the body $L$ is convex for small enough $\varepsilon$. This is a standard perturbation argument; cf. K2, p. 96]. By construction, the body $L$ is obtained as a small perturbation of the Euclidean ball. Since the latter has strictly positive curvature, it is enough to control the first and second derivatives of the function $\epsilon^{k-1}(1-\epsilon)^{-n+k+1}\|x\|_{E}^{-n+k}$. One can see that these are of the order $O\left(\epsilon^{k-3}\right)$, which is small for small enough $\epsilon$ (since $k \geq 4$ ). Therefore, $L$ also has strictly positive curvature.

We now construct $K$, the $k$-intersection body of $L$. If it exists, then by formula (11) we have

where

$$
\|x\|_{K}^{-k}=B_{n, k}\left(\|\cdot\|_{L}^{-n+k}\right)^{\wedge}(x),
$$

see [K2, Lemma 4.5].

$$
B_{n, k}=\frac{k}{(n-k)(2 \pi)^{k}}
$$


Recall that the Fourier transform of $|\cdot|_{2}^{-n+k}, 0<k<n$, equals (see GS, p. $363]$ )

$$
\left(|\cdot|_{2}^{-n+k}\right)^{\wedge}(x)=C_{n, k}|x|_{2}^{-k}
$$

where

$$
C_{n, k}=\frac{2^{k} \pi^{n / 2} \Gamma(k / 2)}{\Gamma((n-k) / 2)} .
$$

In order to compute the Fourier transform for the norms of ellipsoids, we will use the previous formula and the following connection between the Fourier transform and the linear transformations. Let $T$ be an invertible linear transformation on $\mathbb{R}^{n}$; then

Therefore,

$$
\left(|T x|_{2}^{-n+k}\right)^{\wedge}(y)=C_{n, k}|\operatorname{det} T|^{-1}\left|\left(T^{*}\right)^{-1} y\right|_{2}^{-k}
$$

$$
\begin{aligned}
\left(\|\cdot\|_{L}^{-n+k}\right)^{\wedge}(x) & =C_{n, k}\left(|x|_{2}^{-k}-\epsilon^{k}(1-\epsilon)^{k}\left((1-\epsilon)^{2}\left(x_{1}^{2}+\cdots+x_{n-1}^{2}\right)+\epsilon^{2} x_{n}^{2}\right)^{-k / 2}\right) \\
& =C_{n, k}\left(|x|_{2}^{-k}-\left(\frac{x_{1}^{2}+\cdots+x_{n-1}^{2}}{\epsilon^{2}}+\frac{x_{n}^{2}}{(1-\epsilon)^{2}}\right)^{-k / 2}\right) .
\end{aligned}
$$

The latter is strictly positive for all $x \in \mathbb{R}^{n} \backslash\{0\}$. Therefore, the star body $K$ is well defined and is given by

$$
\|x\|_{K}^{-k}=B_{n, k} C_{n, k}\left(|x|_{2}^{-k}-\left(\frac{x_{1}^{2}+\cdots+x_{n-1}^{2}}{\epsilon^{2}}+\frac{x_{n}^{2}}{(1-\epsilon)^{2}}\right)^{-k / 2}\right) .
$$

It remains to show that the body $K$ is not convex. To this end, let us compute the distance from the origin to the boundary of $K$ in the directions $\xi_{1}=(0,0, \ldots, 0,1)$, $\xi_{2}=(\sqrt{2} / 2,0, \ldots, 0, \sqrt{2} / 2)$, and $\xi_{3}=(-\sqrt{2} / 2,0, \ldots, 0, \sqrt{2} / 2)$. One has

$$
\rho_{K}\left(\xi_{1}\right)=\left(B_{n, k} C_{n, k}\right)^{1 / k}\left(1-(1-\epsilon)^{k}\right)^{1 / k},
$$

which can be made as close to zero as we wish by choosing $\epsilon$ sufficiently small.

On the other hand,

$$
\begin{aligned}
\rho_{K}\left(\xi_{2}\right)=\rho_{K}\left(\xi_{3}\right) & =\left(B_{n, k} C_{n, k}\right)^{1 / k}\left(1-\left(\frac{1}{2 \epsilon^{2}}+\frac{1}{2(1-\epsilon)^{2}}\right)^{-k / 2}\right)^{1 / k} \\
& >\left(B_{n, k} C_{n, k}\right)^{1 / k}\left(1-\left(2 \epsilon^{2}\right)^{k / 2}\right)^{1 / k} .
\end{aligned}
$$

The latter does not tend to zero as $\epsilon$ gets small. Thus, the body $K$ is not convex.

Case 2. Let $k=2$. Here $L=L_{\epsilon}$ will be a "smoothened version" of the cube $B_{\infty}^{n}=\left\{x \in \mathbb{R}^{n}: \max _{1 \leq i \leq n}\left|x_{i}\right| \leq 1\right\}$. For simplicity, one can think of $(1-\epsilon) B_{\infty}^{n}+\epsilon B_{2}^{n}$. However, the latter is not a $C^{\infty}$ body. Thus, we will define $L=L_{\epsilon}$ to be an origin-symmetric convex body with $C^{\infty}$ boundary that satisfies the following two conditions:

$$
(1-\epsilon) B_{\infty}^{n} \subset L \subset B_{\infty}^{n}
$$

and

$$
A_{L, e_{1}}(z)=A_{L, e_{1}}(0), \quad \text { for }|z| \leq 1-\epsilon,
$$

where $e_{1}$ is the basis vector $(1,0, \ldots, 0)$. 
We now define a body $K$ as follows:

$$
\|\xi\|_{K}^{-2}=\frac{2}{(n-2)(2 \pi)^{2}}\left(\|\cdot\|_{L}^{-n+2}\right)^{\wedge}(\xi), \quad \xi \in S^{n-1} .
$$

By part (b) of Theorem 2.1 this means

$$
\|\xi\|_{K}^{-2}=-\frac{4}{(2 \pi)^{2}} \int_{0}^{\infty} \frac{A_{L, \xi}(z)-A_{L, \xi}(0)}{z^{2}} d z .
$$

Since the latter integral is strictly negative (and convergent, due to the smoothness and origin-symmetry of the body $L$ ) for every $\xi \in S^{n-1}$, it follows that the star body $K$ is well defined. Moreover, $K$ is the 2-intersection body of $L$.

In order to show that $K$ is not convex, we will compute $\rho_{K}(\xi)$ in the directions $\xi_{1}=(1,0,0, \ldots, 0), \xi_{2}=(\sqrt{2} / 2, \sqrt{2} / 2,0, \ldots, 0)$ and $\xi_{3}=(\sqrt{2} / 2,-\sqrt{2} / 2,0, \ldots, 0)$. By virtue of (3), we have

$$
\begin{gathered}
\rho_{K}^{2}\left(\xi_{1}\right)=\frac{4}{(2 \pi)^{2}} \int_{0}^{\infty} \frac{A_{L, \xi_{1}}(0)-A_{L, \xi_{1}}(z)}{z^{2}} d z=\frac{4}{(2 \pi)^{2}} \int_{1-\epsilon}^{\infty} \frac{A_{L, \xi_{1}}(0)-A_{L, \xi_{1}}(z)}{z^{2}} d z \\
\leq \frac{4 A_{L, \xi_{1}}(0)}{(2 \pi)^{2}} \int_{1-\epsilon}^{\infty} \frac{1}{z^{2}} d z \leq \frac{2^{n+1}}{(2 \pi)^{2}(1-\epsilon)} .
\end{gathered}
$$

Here we used the assumption that $L \subset B_{\infty}^{n}$ and therefore its central sections must not exceed those of the cube: i.e., $A_{L, \xi_{1}}(0) \leq 2^{n-1}$. Thus, $\rho_{K}\left(\xi_{1}\right)$ is bounded above by an absolute constant for all small $\epsilon$.

Now consider

$$
\rho_{K}^{2}\left(\xi_{2}\right)=\frac{4}{(2 \pi)^{2}} \int_{0}^{\infty} \frac{A_{L, \xi_{2}}(0)-A_{L, \xi_{2}}(z)}{z^{2}} d z \geq \frac{4}{(2 \pi)^{2}} \int_{\epsilon}^{\sqrt{2}} \frac{A_{L, \xi_{2}}(0)-A_{L, \xi_{2}}(z)}{z^{2}} d z .
$$

Since $(1-\epsilon) B_{\infty}^{n} \subset L$, we have

$$
A_{L, \xi_{2}}(0) \geq A_{(1-\epsilon) B_{\infty}^{n}, \xi_{2}}(0)=\sqrt{2} 2^{n-1}(1-\epsilon)^{n-1} .
$$

Similarly, $L \subset B_{\infty}^{n}$ implies

$$
A_{L, \xi_{2}}(z) \leq A_{B_{\infty}^{n}, \xi_{2}}(z)=2^{n-1}(\sqrt{2}-z), \quad \text { when }|z| \leq \sqrt{2} .
$$

Thus,

$$
\begin{gathered}
\rho_{K}^{2}\left(\xi_{2}\right) \geq \frac{2^{n+1}}{(2 \pi)^{2}} \int_{\epsilon}^{\sqrt{2}} \frac{\sqrt{2}(1-\epsilon)^{n-1}-(\sqrt{2}-z)}{z^{2}} d z \\
=\frac{2^{n+1}}{(2 \pi)^{2}}\left(\frac{(1-\epsilon)^{n-1}-1}{\epsilon} \sqrt{2}+1-(1-\epsilon)^{n-1}+\ln \sqrt{2}-\ln \epsilon\right) .
\end{gathered}
$$

The latter is large when $\epsilon$ is small.

We also have the same bound for $\rho_{K}^{2}\left(\xi_{3}\right)$. Thus, we have proved that the radius of $K$ can be made as large as we want in the directions $(\sqrt{2} / 2, \sqrt{2} / 2,0, \ldots, 0)$ and $(\sqrt{2} / 2,-\sqrt{2} / 2,0, \ldots, 0)$, while staying bounded in the direction $(1,0,0, \ldots, 0)$. Thus, the body $K$ cannot be convex.

Case 3. Consider $k=3$. For a small $\epsilon>0$ we define an auxiliary origin-symmetric body $M=M_{\epsilon} \subset \mathbb{R}^{n}$ as follows:

$$
M=\left\{x \in \mathbb{R}^{n}: x_{1}^{4}+\epsilon x_{1}^{2}+x_{2}^{2}+x_{3}^{2}+\cdots+x_{n}^{2} \leq 1\right\} .
$$

One can see that the boundary surface of $M$ is obtained by rotating the curve $x_{1}^{4}+\epsilon x_{1}^{2}+x_{2}^{2}=1$ about the $x_{1}$-axis. It is easy to check that this curve has strictly positive curvature. (This can be done by solving for $x_{1}$ in terms of $x_{2}$, as well 
as solving for $x_{2}$ in terms of $x_{1}$, and finding the second derivatives of these two functions.) Therefore, the body $M$ also has strictly positive curvature (and thus is convex).

Now we will compute the parallel section function of $M$ in the direction of the basis vector $e_{1}$.

$$
A_{M, e_{1}}(z)=\kappa_{n-1}\left(1-\epsilon z^{2}-z^{4}\right)^{(n-1) / 2},
$$

where $\kappa_{n-1}$ is the volume of the $(n-1)$-dimensional Euclidean ball $B_{2}^{n-1}$.

Therefore,

$$
A_{M, e_{1}}^{\prime \prime}(0)=-\epsilon(n-1) \kappa_{n-1} \text {. }
$$

We claim that for the body $M_{0}=\left\{x \in \mathbb{R}^{n}: x_{1}^{4}+x_{2}^{2}+x_{3}^{2}+\cdots+x_{n}^{2} \leq 1\right\}$ (which is the limiting case of $M_{\epsilon}$ when $\left.\epsilon \rightarrow 0\right)$ there is a direction $\xi_{0} \notin e_{1}^{\perp}$ such that

$$
A_{M_{0}, \xi_{0}}^{\prime \prime}(0)=-\alpha<0 \text {, }
$$

for some number $\alpha>0$.

Indeed, the body $M_{0}$ has an infinitely smooth norm and therefore (as noted after Theorem 2.1) the Fourier transform $\left(\|\cdot\|_{M_{0}}^{-n+3}\right)^{\wedge}$ is a non-negative continuous function on the sphere. If we had $A_{M_{0}, \xi}^{\prime \prime}(0)=0$ for all $\xi \in S^{n-1}$ outside the equator $e_{1}^{\perp}$, then $\left(\|\cdot\|_{M_{0}}^{-n+3}\right)^{\wedge}$ would be zero on the sphere, and therefore $\|\cdot\|_{M_{0}}^{-n+3}$ would also be identically zero.

Now we will show that $A_{M, \xi_{0}}^{\prime \prime}(0)$ is close to $-\alpha$ when $\epsilon$ is sufficiently small. Since

$$
\left(\|x\|_{M}^{-n+3}\right)^{\wedge}(\xi)=-\pi(n-3) A_{M, \xi}^{\prime \prime}(0),
$$

by part (a) of Theorem 2.1, it is enough to show that $\left(\|x\|_{M}^{-n+3}\right)^{\wedge}\left(\xi_{0}\right)$ is close to $\left(\|x\|_{M_{0}}^{-n+3}\right)^{\wedge}\left(\xi_{0}\right)$ when $\epsilon$ is sufficiently small. One can find explicitly a formula for the norm of $M$ :

$$
\|x\|_{M}=\sqrt{\epsilon x_{1}^{2}+x_{2}^{2}+\cdots+x_{n}^{2}+\sqrt{\left(\epsilon x_{1}^{2}+x_{2}^{2}+\cdots+x_{n}^{2}\right)+4 x_{1}^{4}}} .
$$

Observe that $\|\cdot\|_{M}$ and its first and second derivatives are continuous functions of $(x, \epsilon)$ on $S^{n-1} \times\left[0, \epsilon_{0}\right]$ for some small $\epsilon_{0}$, and are therefore uniformly continuous. Thus, $\|\cdot\|_{M}$ converges to $\|\cdot\|_{M_{0}}$ in $C^{2}\left(S^{n-1}\right)$ as $\epsilon \rightarrow 0$. By [K2, Corollary 3.17] it follows that $\left(\|\cdot\|_{M}^{-n+3}\right)^{\wedge}$ converges to $\left(\|\cdot\|_{M_{0}}^{-n+3}\right)^{\wedge}$ in $C\left(S^{n-1}\right)$.

Thus, for small enough $\epsilon$ we have that $\left(\|\cdot\|_{M}^{-n+3}\right)^{\wedge}\left(\xi_{0}\right)$ is close to $\pi(n-3) \alpha$ and $\left(\|\cdot\|_{M}^{-n+3}\right)^{\wedge}\left(e_{1}\right)$ is close to zero. We now fix $\epsilon$ so small that

$$
\left(\|\cdot\|_{M}^{-n+3}\right)^{\wedge}\left(\xi_{0}\right)>\left|\left\langle\xi_{0}, e_{1}\right\rangle\right|^{-3}\left(\|\cdot\|_{M}^{-n+3}\right)^{\wedge}\left(e_{1}\right) .
$$

For a small $\lambda>0$ define an origin-symmetric body $L$ as follows:

$$
\|x\|_{L}^{-n+3}=\|x\|_{M}^{-n+3}+\lambda|x|_{2}^{n+3} .
$$

Since $M$ has strictly positive curvature, a small perturbation will not affect this property. Thus $L$ is convex for small enough $\lambda$. We will also require that $\lambda$ be small enough to guarantee that

$$
\begin{aligned}
\left(\|\cdot\|_{M}^{-n+3}\right)^{\wedge}\left(\xi_{0}\right)+\lambda(\mid & \left.\left.\cdot\right|_{2} ^{-n+3}\right)^{\wedge}\left(\xi_{0}\right) \\
& >\left|\left\langle\xi_{0}, e_{1}\right\rangle\right|^{-3}\left(\left(\|\cdot\|_{M}^{-n+3}\right)^{\wedge}\left(e_{1}\right)+\lambda\left(|\cdot|_{2}^{-n+3}\right)^{\wedge}\left(e_{1}\right)\right) .
\end{aligned}
$$


Now define a star body $K$ as follows:

$$
\|x\|_{K}^{-3}=\frac{3}{(n-3)(2 \pi)^{3}}\left(\|\cdot\|_{L}^{-n+3}\right)^{\wedge}(x), \quad x \in \mathbb{R}^{n} \backslash\{0\} .
$$

Since $\left(\|\cdot\|_{M}^{-n+3}\right)^{\wedge}(x) \geq 0$ and $\left(|\cdot|_{2}^{-n+3}\right)^{\wedge}(x)>0$ for all $x \in \mathbb{R}^{n} \backslash\{0\}$, it follows that

$$
\left(\|\cdot\|_{L}^{-n+3}\right)^{\wedge}(x)=\left(\|\cdot\|_{M}^{-n+3}\right)^{\wedge}(x)+\lambda\left(|\cdot|_{2}^{-n+3}\right)^{\wedge}(x)>0, \quad x \in \mathbb{R}^{n} \backslash\{0\},
$$

and therefore the star body $K$ is well defined. Also observe that $K$ is the 3 intersection body of $L$.

Finally we show that $K$ is not convex. First, note that $K$ is a body of revolution about the $x_{1}$-axis, since $M$ was such. Secondly, condition (4) implies that

$$
\left|\left\langle\xi_{0}, e_{1}\right\rangle\right| \rho_{K}\left(\xi_{0}\right)>\rho_{K}\left(e_{1}\right) .
$$

This means that the projection of the vector $\rho_{K}\left(\xi_{0}\right) \xi_{0} \in K$ onto the axis of revolution of $K$ lies outside of $K$. Thus, $K$ is not convex.

\section{REFERENCES}

[B] Gautier Berck, Convexity of $L_{p}$-intersection bodies, Adv. Math. 222 (2009), no. 3, 920936, DOI 10.1016/j.aim.2009.05.009. MR.2553373 (2010m:52008)

[BZ] Jean Bourgain and Gaoyong Zhang, On a generalization of the Busemann-Petty problem, Convex geometric analysis (Berkeley, CA, 1996), Math. Sci. Res. Inst. Publ., vol. 34, Cambridge Univ. Press, Cambridge, 1999, pp. 65-76. MR1665578 (99m:52011)

[G] Richard J. Gardner, Geometric tomography, 2nd ed., Encyclopedia of Mathematics and its Applications, vol. 58, Cambridge University Press, Cambridge, 2006. MR 2251886 (2007i:52010)

[GG] R. J. Gardner and A. A. Giannopoulos, p-cross-section bodies, Indiana Univ. Math. J. 48 (1999), no. 2, 593-613, DOI 10.1512/iumj.1999.48.1689. MR1722809 (2000i:52002)

[GKS] R. J. Gardner, A. Koldobsky, and T. Schlumprecht, An analytic solution to the Busemann-Petty problem on sections of convex bodies, Ann. of Math. (2) 149 (1999), no. 2, 691-703, DOI 10.2307/120978. MR1689343(2001b:52011)

[GS] I. M. Gel'fand and G. E. Shilov, Generalized functions. Vol. 1, Properties and operations, Academic Press [Harcourt Brace Jovanovich Publishers], New York, 1964 [1977]. Translated from the Russian by Eugene Saletan. MR0435831 (55 \#8786a)

[H] Christoph Haberl, $L_{p}$ intersection bodies, Adv. Math. 217 (2008), no. 6, 2599-2624, DOI 10.1016/j.aim.2007.11.013. MR2397461 (2009a:52001)

[HL] Christoph Haberl and Monika Ludwig, A characterization of $L_{p}$ intersection bodies, Int. Math. Res. Not., posted on 2006, Art. ID 10548, 29, DOI 10.1155/IMRN/2006/10548. MR2250020 (2007k:52007)

[K1] A. Koldobsky, A functional analytic approach to intersection bodies, Geom. Funct. Anal. 10 (2000), no. 6, 1507-1526, DOI 10.1007/PL00001659. MR:1810751(2001m:52007)

[K2] Alexander Koldobsky, Fourier analysis in convex geometry, Mathematical Surveys and Monographs, vol. 116, American Mathematical Society, Providence, RI, 2005. MR2132704 (2006a:42007)

[KKZ] A. Koldobsky, H. König, and M. Zymonopoulou, The complex Busemann-Petty problem on sections of convex bodies, Adv. Math. 218 (2008), no. 2, 352-367, DOI 10.1016/j.aim.2007.12.006. MR2407938 (2009c:52016)

[KPZ1] A. Koldobsky, G. Paouris, and M. Zymonopoulou, Isomorphic properties of intersection bodies, J. Funct. Anal. 261 (2011), no. 9, 2697-2716, DOI 10.1016/j.jfa.2011.07.011. MR2826412 (2012j:52010)

[KPZ2] A. Koldobsky, G. Paouris, and M. Zymonopoulou, Complex intersection bodies, J. Lond. Math. Soc. (2) 88 (2013), no. 2, 538-562, DOI 10.1112/jlms/jdt014. MR3106735

[KY] Alexander Koldobsky and Vladyslav Yaskin, The interface between convex geometry and harmonic analysis, CBMS Regional Conference Series in Mathematics, vol. 108, Published for the Conference Board of the Mathematical Sciences, Washington, DC, by the American Mathematical Society, Providence, RI, 2008. MR2365157 (2008j:52008) 
[KYZ] Jaegil Kim, Vladyslav Yaskin, and Artem Zvavitch, The geometry of p-convex intersection bodies, Adv. Math. 226 (2011), no. 6, 5320-5337, DOI 10.1016/j.aim.2011.01.011. MR2775903 (2012e:44002)

[L] Erwin Lutwak, Intersection bodies and dual mixed volumes, Adv. in Math. 71 (1988), no. 2, 232-261, DOI 10.1016/0001-8708(88)90077-1. MR963487 (90a:52023)

[LYZ] Erwin Lutwak, Deane Yang, and Gaoyong Zhang, $L_{p}$ affine isoperimetric inequalities, J. Differential Geom. 56 (2000), no. 1, 111-132. MR.1863023(2002h:52011)

[LZ] Erwin Lutwak and Gaoyong Zhang, Blaschke-Santaló inequalities, J. Differential Geom. 47 (1997), no. 1, 1-16. MR.1601426 (2000c:52011)

[M1] Emanuel Milman, Generalized intersection bodies, J. Funct. Anal. 240 (2006), no. 2, 530-567, DOI 10.1016/j.jfa.2006.04.004. MR2261694 (2007h:52007)

[M2] Emanuel Milman, Generalized intersection bodies are not equivalent, Adv. Math. 217 (2008), no. 6, 2822-2840, DOI 10.1016/j.aim.2007.11.007. MR2397468(2009k:52012)

[MP] V. D. Milman and A. Pajor, Isotropic position and inertia ellipsoids and zonoids of the unit ball of a normed n-dimensional space, Geometric aspects of functional analysis (1987-88), Lecture Notes in Math., vol. 1376, Springer, Berlin, 1989, pp. 64-104, DOI 10.1007/BFb0090049. MR1008717 (90g:52003)

[S] Jared Schlieper, A note on k-intersection bodies, Proc. Amer. Math. Soc. 135 (2007), no. 7, 2081-2088 (electronic), DOI 10.1090/S0002-9939-07-08774-6. MR2299484 (2008d:52011)

[Y] Vladyslav Yaskin, On strict inclusions in hierarchies of convex bodies, Proc. Amer. Math. Soc. 136 (2008), no. 9, 3281-3291, DOI 10.1090/S0002-9939-08-09424-0. MR2407094 (2009h:52010)

[YY] V. Yaskin and M. Yaskina, Centroid bodies and comparison of volumes, Indiana Univ. Math. J. 55 (2006), no. 3, 1175-1194, DOI 10.1512/iumj.2006.55.2761. MR2244603 (2007f:52008)

[Z1] Gaoyong Zhang, Sections of convex bodies, Amer. J. Math. 118 (1996), no. 2, 319-340. MR.1385280 (97f:52015)

[Z2] Gaoyong Zhang, A positive solution to the Busemann-Petty problem in $\mathbf{R}^{4}$, Ann. of Math. (2) 149 (1999), no. 2, 535-543, DOI 10.2307/120974. MR.1689339 (2001b:52010)

Department of Mathematical and Statistical Sciences, University of Alberta, Edmonton, Alberta T6G 2G1, Canada

E-mail address: vladyaskin@math.ualberta.ca 